\title{
Review of Experimental Techniques used to Study the Mechanical Behaviour of Biological Soft Tissues
}

\author{
S. Kadhane ${ }^{1 *}$, H. Warhatkar ${ }^{2}$ \\ ${ }^{1}$ Research Scholar, ${ }^{2}$ Associate Professor \\ Dr.Babasaheb Ambedkar Technological University, Lonere, Raigad, India \\ \{shkadhane@gmail.com\}
}

\begin{abstract}
This review is intended to highlight and discuss the various experimental techniques used to obtain the mechanical behavior of soft tissues at varying strain rates. A variety of techniques used to obtain the mechanical properties of soft tissues and soft materials from low to intermediate and high rates of strain are summarized. These techniques include quasi-static, intermediate and dynamic strain rate setups. Split Hopkinson Pressure Bar (SHPB) technique with some modifications is commonly used for testing soft materials at high strain rates up to $10^{4} \mathrm{~s}^{-1}$. Article searches were performed on impact biomechanics, orthopaedic and biomechanical publications in databases: PubMed, Scopus, ScienceDirect, Compendex, MEDLINE and EMBASE. The discrepancies on the use of conventional SHPB in the dynamic soft tissues testing were highlighted. This review explains the use of conventional SHPB technique for the characterization of soft tissues under compressive and tensile loading. The discrepancies in existing literature emphasize the need for additional research into the development of SHPB for dynamic testing of soft tissues under both compressive and tensile loading. This review suggests requirement of an integrated SHPB setup with necessary modifications in striker bar loading mechanism and specimen clamping attachment for dynamic testing of soft tissues under compressive as well as tensile loading.
\end{abstract}

Keywords: SHPB, soft tissue properties, human body modelling, high strain rates, numerical simulations

\section{Introduction}

The various events subjected to dynamicloading include automotive crash testing, high speed automotive accidents, ballistic and blast injuries, aircraft impacts and high impact sports activities such as football, athletic games. The performance of soft and hard tissues at static and dynamic conditions in these events is strain rate dependant. Human body finite element modeling requires strain rate dependant properties of soft materials. Finite element models of human body are most useful in designing the safer vehicles by conducting crash tests. The material behavior is important to understand the injury patterns during pedestrian - vehicle high speed impacts. A review has been conducted for the available material properties and dynamic behavior of soft tissues extracted from animal and human body. The human body is divided into three parts viz. a) Lower extremityKnee, Thigh, Hip b) Head, neck and spine c) Upper extremity, chest and abdomen. The material property data in the literature was reported on the soft tissues such as ligaments, tendons, cartilage, meniscus, brain tissue, skin, muscle, fat-white adipose tissue.

An impact occurring at sufficiently large velocities (at high strain rates) causes inelastic and particularly plastic deformations. These plastic and elastic deformations may cause large strains. Unfortunately, dependence of stress on the strain, strain rate for various engineering materials and biological materials is still not understood. Thus, the various experimental methods were developed to understand the properties of materials at high rates of strain. The machines for obtaining mechanical data at higher rates of strain have been developed historically in the various research laboratories and some companies which include SHPB, miniaturized SHPB and plate impact facilities. The strain rate regime and testing methodology used to investigate rate dependant materials properties is tabulated in Table 1 .

B. Iyer, S. Nalbalwar and R. Pawade (Eds.)

ICCASP/ICMMD-2016. Advances in Intelligent Systems Research.

Vol. 137, Pp. 277-283.

(c) 2017. The authors - Published by Atlantis Press

This is an open access article under the CC BY-NC license (http://creativecommons.org/licens)es/by-nc/4.0/). 
Table 1 Strain rate testing domains [27-28]

\begin{tabular}{|l|l|l|l|}
\hline $\begin{array}{l}\text { Loading Time } \\
(\mathbf{s})\end{array}$ & $\begin{array}{l}\text { Strain Rate } \\
\text { Regime }\left(\mathbf{S}^{-1}\right)\end{array}$ & Testing Domain & Testing Methodology \\
\hline $10^{6}-10^{4}$ & $<10^{-6} \mathrm{~s}^{-1}$ & Creep domain & Constant load machines \\
\hline $10^{4}-10^{2}$ & $10^{-3} \mathrm{~s}^{-1}-10^{-1} \mathrm{~s}^{-1}$ & Quasi static & Servo hydraulic machines \\
\hline $10^{0}-10^{-1}$ & $10^{0} \mathrm{~s}^{-1}-10^{2} \mathrm{~s}^{-1}$ & Intermediate strain rate & Specialized machines \\
\hline $10^{-2}-10^{-4}$ & $10^{2} \mathrm{~s}^{-1}-10^{4} \mathrm{~s}^{-1}$ & High strain rate & SHPB technique \\
\hline $10^{-4}-10^{-6}$ & $10^{4} \mathrm{~s}^{-1}-10^{6} \mathrm{~s}^{-1}$ & Very high strain rate & Miniaturized Kolsky bar \\
\hline$>10^{-6}$ & $>10^{6} \mathrm{~s}^{-1}$ & Ultra high strain rate & Explosive driven plate impact \\
\hline
\end{tabular}

\section{Experimental Testing Methods}

The available research papers have been reviewed to understand the various experimental methods from quasistatic to medium and high strain rates which are used to characterize the dynamic behavior of biological soft tissues.

\subsection{Quasi-static testing}

Quasi-static tests have been performed by using standard universal testing machines [1-11] for testing porcine muscle [1], bovine brain tissue [2], bovine kidney tissue [3], porcine adipose tissue [4], polyurea elastomer [5], gelatin tissue simulant [6] under compressive loading, while bovine tendon [7], pig skin [8], human cadaveric gastro-colic ligament [9], EPDM soft rubber [10], and polymer RTM-6 soft material [11] under tensile loading.

The most of the studies on biological soft tissues, tissue simulants and soft materials in existing literature have been focused on test conducted at near-constant strain rates. This is preliminary due to experimental difficulties associated with dynamic testing of soft tissues. The limitation of quasi-static setup is an accurate measurement of small applied forces. Quasi-static test gives load-displacement data, which is then converted into stress-strain data using the specimen dimensions. Quasi-static properties indicate hyperelasticity and almost strain rate dependency.

\subsection{Intermediate strain rate testing}

Intermediate strain rate experiments have been conducted by using custom built or drop tower test setups [3-5] for testing bovine kidney tissue [3], porcine adipose tissue [4] and polyurea [5] under compressive loading conditions. The mechanical response in the intermediate strain rate range is a much less explored range for any material, including soft materials and soft tissues. Inter-mediate strain rate testing of soft tissues is conducted using custom built or drop weight test setup. These setups do not give directly whole stress-strain curve in terms of strain rate. Thus, it is essential to develop most reliable and flexible experimental methods to illustrate mechanical behaviour of soft materials at different intermediate rates of strain.

\subsection{High Strain Rate Testing using SHPB}

J. Hopkinson [23] initially conducted rupture test of iron wire by the impact of drop weight in 1872. Later on Bertram Hopkinson [24] invented pressure bars to measure pressure produced by high explosives or high speed impacts of bullets in 1914. The compression version of SHPB is initially developed by H. Kolsky in 1949 [25] 
to measure dynamic stress - strain response under high velocity impact loading condition using condenser microphones. Krafft et al. in 1954 used strain gauge in the Kolsky bar to measure and analyze the stress waves, which become a standard experimental measurement method for Kolsky bar. The Kolsky bar used under compressive loading is also called as Split Hopkinson Pressure Bar in the memory of John Hopkinson and his son Bertram Hopkinson. Dynamic experiments have been performed by using various modified versions of SHPBs to understand the high rate behavior of human cadaveric body lower extremity muscles [14] and liver tissues [15], bovine muscles [16], porcine muscles [1], bovine kidney tissue [3], porcine white adipose tissue [4], porcine trachea [17], EPDM rubber [10], polyurea [5] and gelatin bio-material [6] under dynamic compressive loading conditions, while bovine tendon [7], pig skin [8], human skin [18-19], human ligaments from cervical spine [20], soft polymer [11], EPDM rubber [10] under tensile loading conditions. Bovine brain tissue has been tested under torsional loading using modified Kolsky bar [21] to obtain shear response.

This review summarizes important issues related with the application of SHPB in the characterization of soft tissues and soft materials. These issues are weak transmitted signals, constant rate of deformation, uniform loading on the specimen, specimen gripping methods and inertia effects. The possible SHPB modifications along with the gaps in literature are summarized to find the discrepancies in available literature. The variations in the reported material properties and dynamic behavior have been used for further design and development of experimental testing setup and development of alternate material models. Various researchers have offered some developments in SHPB and are summarized in Table 2.

Table 2 Recent developments in SHPB testing of soft materials

\begin{tabular}{|l|l|}
\hline Year & Development in SHPB \\
\hline $1994-1997$ & Use of viscoelastic pressure bars [16], [34], [40], [41], [42] \\
\hline 2006 & Wave attenuation and dispersion [14], [1], [10], [27], [42] \\
\hline 2007 & $\begin{array}{l}\text { Use of quartz crystal and semiconductor strain gauges, Minimization of } \\
\text { inertia effect [10], [14] }\end{array}$ \\
\hline 2009 & $\begin{array}{l}\text { Use of hollow transmission bar, Pulse shaping technique and Momentum } \\
\text { trapping mechanism [2-9], [39] }\end{array}$ \\
\hline 2009 & Minimization in duration of incident pulse [5] \\
\hline 2015 & Minimization of effect of friction [44] \\
\hline
\end{tabular}

The possible developments includes the use of viscoelastic pressure bars, implementation of wave correction, minimization of frictional and inertial effects, use of hollow transmission bars and quartz crystal. These modifications need to be implemented in SHPB experimentation to study the wide range of soft materials and soft tissues for impact modeling but assumptions used in deriving the Equations (1) to (3) are suspect for these modifications.

\section{SHPB Theory}

The most commonly used method to determine the dynamic material properties is the Split Hopkinson Pressure Bar (SHPB) apparatus. It is used to predict the stress - strain response of engineering materials at high rates of strain between $10^{2}$ to $10^{4} \mathrm{~s}^{-1}$. SHPB design consists of long steel bar, material specimen and a ballistic pendulum. High velocity impact was generated by hitting the steel bar through an explosive charge. A compressive wave generated due to impact would travel through the long bar into the test specimen. The pressures travelled through the long bar would be similar to pressures seen during an impact. Hopkinson was able to generate pressure-time curves from experiments that would describe an impact event. 
In 1949, H. Kolsky added a second long pressure bar to original design developed by Hopkinson. He sandwiched the test specimen in between the pressure bars instead of keeping a specimen at a long distance from one end of bar. Due to this, Hopkins pressure bar got the name as Split- Hopkinson Pressure Bar. This design became most popular and extensively used to establish dynamic properties of engineering materials. The original SHPB was designed to understand dynamic compressive behavior of an engineering material. But, other Hopkinson Bar schemes has been devised to test specimens under tensile and torsional loading. The purpose of SHPB testing is to obtain high rate dynamic material properties under compression, tension as well as in torsional mode. Although there is no worldwide standard design is available for the SHPB apparatus, as all SHPB share common elements.

\subsection{Direct compressive and tensile SHPB apparatus}

A schematic of direct compressive SHPB and tensile SHPB used to obtain dynamic compressive and tensile material properties is shown in Fig. 1 and Fig. 2 respectively.

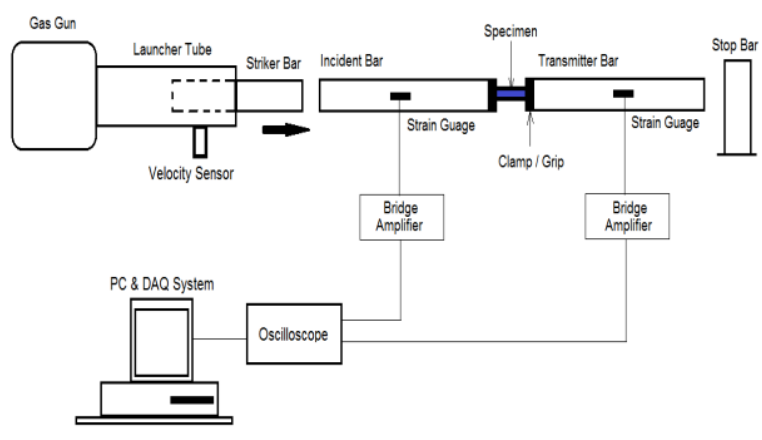

Fig. 1. Direct Compressive SHPB[27-28]

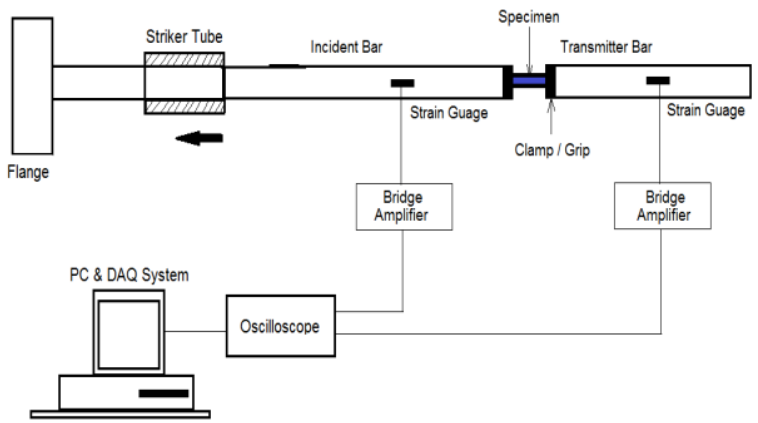

Fig. 2. Direct Tensile SHPB[27-28]

In compressive SHPB, the cylindrical test specimen is kept between incident and transmitter bar by means of an adhesive. An elastic stress pulse compressive in nature is generated by making an impact of compressive solid striker bar on the incident bar.

Similarly, in tensile SHPB, the hollow tensile specimen is kept between incident and transmitter by means of clamps. An elastic stress pulse tensile in nature is generated by making impact of tensile hollow striker bar on the collar attached to the incident bar. The amplitude and magnitude of stress wave pulses generated due to impact are measured by mounting the strain gauges on the pressure bars.

One dimensional wave propagation theory in elastic pressure bars along axial direction yields the calculation of strain $\varepsilon_{s}$, strain rate $\dot{\varepsilon_{s}}$ and stress $\sigma_{s}$ induced in the test specimen using following set of equations.

$$
\begin{aligned}
& \varepsilon_{s}=\frac{-2 C_{0}}{L_{s}} \int_{0}^{t} \varepsilon_{r} d t \\
& \dot{\varepsilon}_{s}=\frac{-2 C_{0}}{L_{s}} \varepsilon_{r} \\
& \sigma_{s}=\frac{A_{0}}{A_{s}} E \varepsilon_{t}
\end{aligned}
$$

Bacon's experimental approach [26] has been used to calculate the wave attenuation and propagation coefficients of viscoelastic pressure bars. The calculated propagation coefficients are used during the processing of actual specimen experimental test data to work out the specimen stress-strain behavior. Thus, transmitted and reflected pulses need to be reconstructed by using attenuation and dispersion correction in the measured pulses 
for the distance by which it has propagated. The calculation of specimen stress, strain and strain rate for polymeric bars in the frequency domain was done using viscoelastic analysis, where the phase velocity is a function of frequency. Then, strain $\varepsilon_{s}(t)$, strain rate $\dot{\varepsilon}_{s}(t)$ and stress $\sigma_{s}(t)$, in the test specimen can be calculated in time domain using inverse Fourier transform.

\subsection{Proposed integrated polymeric SHPB}

The conventional SHPB setup is a familiar device used for the characterization of high strain-rate behavior of engineering material under dynamic compressive loading. The various researchers used different SHPB methods for tensile testing of soft material and all are based on compressive experiments. Therefore, it would be convenient to simply have one system, which is competent to take measurements both in compressive and tensile loading. The various challenges encountered in the design and development of SHPB setup are compressive and tensile loading mechanism, specimen grip, dynamic stress-equilibrium in specimen and the measurement of low weak transmitted signal. The conventional SHPB is used to investigate the strain rate dependant compressive behavior of elastic. This method with various modifications has been used for dynamic characterization of soft materials and soft tissues under compressive, tensile and torsional loading conditions. The soft tissues like muscle and skin as well as soft materials like foam should to be tested along transverse and longitudinal fiber orientation under tensile and compressive loadings when subjected to complex loading condition. Hence, it is proposed to design and develop an integrated polymeric SHPB setup which is capable of testing soft tissues under both compressive and tensile loading at variable rates of strain. The main goal in designing integrated polymeric SHPB setup is to find a way to convert a compressive SHPB setup into a tensile SHPB setup with minimum changes possible in order to easily go back and forth between the two systems. The various challenges encountered in the design and development of an integrated polymeric SHPB setup are compressive and tensile loading mechanism, specimen gripping, dynamic stress equilibrium, the measurement of low weak transmitted signal and wave attenuation and dispersion.

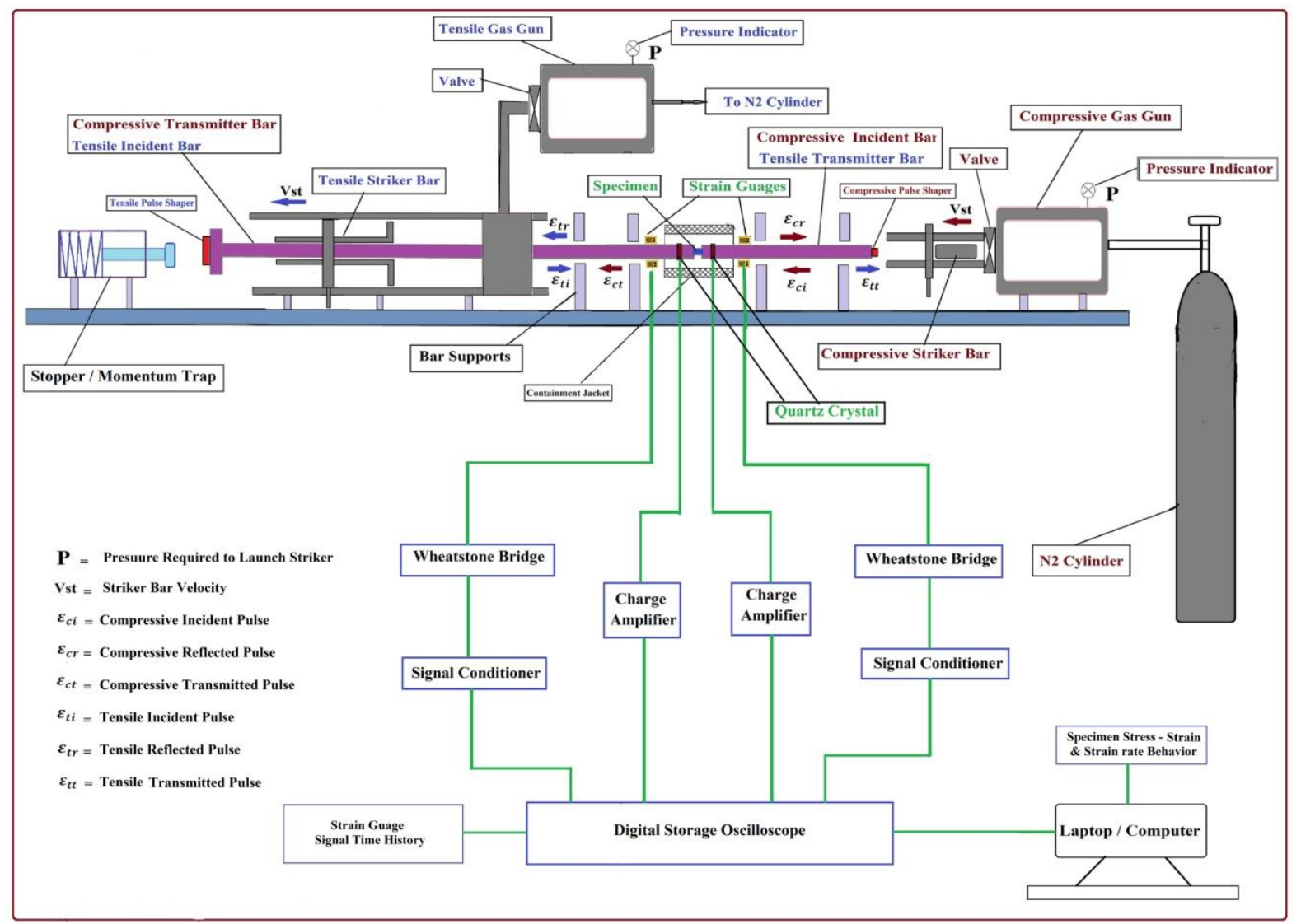

Fig. 3. Working block diagram of proposed integrated PSHPB setup

The self explanatory working block diagram of proposed integrated polymeric SHPB setup is depicted in Fig. 3. It consists of polymeric loading bar technique and two-stage air operated striker bar launching mechanism for both compressive and tensile tests. It would therefore be possible to take measurements on soft tissues both in 
compressive and tensile loading using single SHPB setup by changing the specimen holding and striker loading attachment. A traditional digital storage oscilloscope assisted data acquisition system is established to acquire the stress pulses using strain gauges and Matlab program is used for post processing of the recorded data to plot the stress-strain response at each strain rate. IR sensor based velocity measurement system is designed to record an experimental impact velocity of the striker bar for calibration of theoretical velocity of striker bar.

In compressive polymeric SHPB, the cylindrical specimen is sandwiched between incident and transmitter bar by means of an adhesive and compressive stress pulse is generated by the impact of compressive solid striker bar on the end of incident bar. Similarly, in tensile polymeric SHPB, the hollow tensile specimen is clamped between incident and transmitter by means of clamps and tensile elastic stress pulse is generated by the impact of tensile hollow striker bar on the collar attached to the incident bar. The data acquisition system and Matlab analysis program will remains same for both compressive and tensile loading condition except the specimen gripper and loading attachment. The specimen preparation procedure for both compression and tension test is different as specimens has different size and shape.

\section{Conclusions}

The various experimental methods for impact testing of soft tissues were reported. Quasi-static and intermediate strain rate testing of various soft tissues were studied. The conventional SHPB technique needs to be modified for dynamic testing of soft tissues. The discrepancies in available literature of SHPB testing of soft tissues highlight the need for further research into the development of SHPB testing [1, 2, 10, 16, and 44]. This review suggests requirement of an integrated SHPB setup with necessary modifications in striker bar loading mechanism and specimen clamping attachment for dynamic testing of soft tissues under compressive as well as tensile loading.

\section{Acknowledgement}

The authors would like to thank TEQIP-II, Government of India and DBATU, Lonere for providing the financial support to purchase various instruments and software's required to develop an experimental and numerical testing facility during this research work.

\section{References}

[1]. Bo Song, Weinong Chen, Yun Ge and Tusit Weerasooriya, Dynamic and quasi-static compressive response of porcine muscle, Journal of Biomechanics, 40 (2007) 2999-3005.

[2]. Farhana Pervin and Weinong W. Chen, Dynamic mechanical response of bovine gray and white matter brain tissues under compression, Journal of Biomechanics, 42 (2009) 731-735.

[3]. Farhana Pervin, Weinong W. Chen and Tusit Weerasooriya, Dynamic compressive response of renal cortex, International Journal of Structural Changes in Solids, 2(1) (2010) 1 - 7.

[4]. Kerstyn Comley and Norman Fleck, The compressive response of porcine adipose tissue from low to high strain rate, International Journal of Impact Engineering, 46 (2010) 1 - 10.

[5]. Jongmin Shim and Dirk Mohr, Using Split Hopkinson pressure bars to perform large strain compression tests on polyurea at low, intermediate and high strain rates, International Journal of Impact Engineering, 36 (2009) 1116-1127.

[6]. Jiwoon Kwon and Ghatu Subhas, Compressive strain rate sensitivity of ballistic gelatin, Journal of Biomechanics, 43 (2010), 420-425.

[7]. Ming Cheng, Weinong Chen and Tusit Weerasooriya, Mechanical behavior of bovine tendon with stress and loading rate effects, Advanced Theoretical Applied Biomechanics, 2(2) (2009) 59 - 74.

[8]. Jaeyoung Lim, Jihey Hong, Weinong W. Chen, Tusit Weerasooriya, Mechanical response of pig skin under dynamic tensile testing, International Journal of Impact Engineering, 38 (2011) 130 - 135. 
[9]. O. Chebil, M. Behr and B.J. Arnoux, Quasi-static failure properties of human gastro-colic ligament, Computer methods in Biomechanics and Biomedical engineering, Taylor and Francis,15(1) (2012) 283 $-284$.

[10]. B. Song and W. Chen, One-dimensional dynamic compressive behavior of EPDM rubber, Transactions of ASME, 125 (2003) 294-301.

[11]. Robert Gerlach, Clive R. Siviour, Nik Petrnic, Jens Wiegand, Experimental characterization and constitutive modeling of RTM-6 resin under impact loading, Polymer Testing, 49 (2011) 2728 - 2737.

[12]. A Chawla, S Mukherjee, H Warhatkar, R Malhotra, Dynamic characterization of bovine medial Collateral ligaments, IRCOBI Conference Proceedings. International Research Council on the Biomechanics of Injury, York, (2009) 109-113.

[13]. Hemant Warhatkar, Anoop Chawla, Sudipto Mukherjee, Rajesh Malhotra, Experimental Study of Variation between Quasi-Static and Dynamic Load Deformation Properties of Medial Collateral Knee Ligaments, SAE World Congress, Detriot, (2009).

[14]. Karthikeyan Balaraman, Sudipto Mukherjee, Anoop Chawla and Rajesh Malhotra, Dynamic compressive response of passive human muscles using Split Hopkinson Pressure Bar, Indian Journal of Biomechanics, 1.3 (2012) 20-28.

[15]. H. Saraf, K.T. Ramesh, A.M. Lennon, A.C. Merkle, Mechanical properties of soft human tissues under dynamic loading, Journal of Biomechanics, 40 (2007) 1960-1967.

[16]. Caleb Van Sligtenhorst, Duane S. Cronin, G. Wayne Brodland, High strain rate compressive properties of bovine muscle tissue determined using split Hopkinson bar apparatus, Journal of Biomechanics, 39 (2006) 1852-1858.

[17]. B J Butler, C Bo, A W Tucker, A P Jardine, W G Proud, A Williams and K A Brown, Mechanical and histological characterization of trachea tissue subjected to blast-type pressures, Journal of Physics: Conference Series, 18th APS-SCCM and 24th AIRAPT, IOP, 500 (2014). 